\title{
Optimization of disassembly line balancing using an improved multi-objective Genetic Algorithm
}

\author{
Wang, Y.J. ${ }^{\text {a, }}$, Wang, N.D. ${ }^{a}$, Cheng, S.M. ${ }^{a}$, Zhang, X.C. ${ }^{a}$, Liu, H.Y. ${ }^{a}$, Shi, J.L. ${ }^{a}$, Ma, Q.Y. ${ }^{a}$, Zhou, M.J. ${ }^{a}$ \\ ${ }^{\mathrm{a} S}$ School of Mechanical Engineering and Automation, Dalian Polytechnic University, P.R. China
}

\begin{abstract}
A B S T R A C T
Disassembly activities take place in various recovery operations including remanufacturing, recycling, and disposal. Product disassembly is an effective way to recycle waste products, and it is a necessary condition to make the product life cycle complete. According to the characteristics of the product disassembly line, based on minimizing the number of workstations and balancing the idle time in the station, the harmful index, the demand index, and the number of direction changes are proposed as new optimization objectives. So based on the analysis of the traditional genetic algorithm into the precocious phenomenon, this paper constructed the multi-objective relationship of the disassembly line balance problem. The disassembly line balance problem belongs to the NP-hard problem, and the intelligent optimization algorithm shows excellent performance in solving this problem. Considering the characteristics of the traditional method solving the multi-objective disassembly line balance problem that the solution result was single and could not meet many objectives of balance, a multi-objective improved genetic algorithm was proposed to solve the model. The algorithm speeds up the convergence speed of the algorithm. Based on the example of the basic disassembly task, by comparing with the existing single objective heuristic algorithm, the multiobjective improved genetic algorithm was verified to be effective and feasible, and it was applied to the actual disassembly example to obtain the balance optimization scheme. Two case studies are given: a disassembly process of the automobile engine and a disassembly of the computer components.
\end{abstract}

\begin{tabular}{l} 
A R T I C L E I N F O \\
\hline Keywords: \\
Assembly; \\
Disassembly; \\
Line balancing; \\
Multi-objective optimization; \\
Remanufacturing; \\
Product recovery; \\
Product life cycle; \\
NP-hard problem; \\
Improved genetic algorithm \\
*Corresponding author: \\
wangyj@dlpu.edu.cn \\
(Wang, Y.J.) \\
Article history: \\
Received 13 May 2021 \\
Revised 23 May 2021 \\
Accepted 4 June 2021 \\
$\begin{array}{l}\text { CC) (i) } \\
\text { BY }\end{array}$ \\
Content from this work may be used under the terms of \\
the Creative Commons Attribution 4.0 International \\
Licence (CC BY 4.0.). Any further distribution of this work \\
must maintain attribution to the author(s) and the title of \\
the work, journal citation and DoI.
\end{tabular}

\section{References}

[1] Güngör, A., Gupta, S.M. (2002). Disassembly line in product recovery, International Journal Production Research, Vol. 40, No. 11, 2569-2589, doi: 10.1080/00207540210135622.

[2] Avikal, S., Mishra, P.K., Jain, R. (2014). A fuzzy AHP and PROMETHEE method-based heuristic for disassembly line balancing problems, International Journal of Production Research, Vol. 52, No. 5, 1306-1317, doi: 10.1080/ 00207543.2013.831999.

[3] Altekin, F.T., Akkan, C. (2012). Task-failure-driven rebalancing of disassembly lines, International Journal of Production Research, Vol. 50, No. 18, 4955-4976, doi: 10.1080/00207543.2011.616915.

[4] Kalayci, C.B., Gupta, S.M. (2011). A hybrid genetic algorithm approach for disassembly line balancing, In: Proceedings of the 42 ${ }^{\text {nd }}$ Annual Meeting of Decision Science Institute, Boston, Massachusetts, USA, 2142-2148.

[5] Kalayci, C.B., Gupta, S.M., Nakashima, K. (2011). A simulated annealing algorithm for balancing a disassembly line, In: Matsumoto, M., Umeda, Y., Masui, K., Fukushige, S. (eds), Design for innovative value towards a sustainable society, Springer-Verlag, Berlin, Germany, 714-719, doi: 10.1007/978-94-007-3010-6 143.

[6] Wu, W., Liu, W., Zhang, F., Dixit, V. (2021). A new flexible parking reservation scheme for the morning commute under limited parking supplies, Networks and Spatial Economics, In press, 2021, doi: 10.1007/s11067-021$\underline{09538-5}$ 
[7] Palakodati, S.S.S., Chirra, V.R., Dasari, Y., Bulla, S. (2020). Fresh and rotten fruits classification using CNN and transfer learning, Revue d'Intelligence Artificielle, Vol. 34, 617-622, doi: 10.18280/ria.340512.

[8] Xiao, Y., Li, C., Song, L., Yang, J., Su, J. (2021). A multidimensional information fusion-based matching decision method for manufacturing service resource, IEEE Access, Vol. 9, 39839-39851, doi: 10.1109/ACCESS.2021. $\underline{3063277 .}$.

[9] Pilati, F., Ferrari, E., Gamberi, M., Margelli, S. (2021). Multi-manned assembly line balancing: workforce synchronization for big data sets through simulated annealing, Applied Science, Vol. 11, No. 6, Article No. 2523, doi: $10.3390 /$ app11062523.

[10] Chutima, P., Suchanun, T. (2019). Productivity improvement with parallel adjacent U-shaped assembly lines, Advances in Production Engineering \& Management, Vol. 14, No. 1, 51-64, doi: 10.14743/apem2019.1.311.

[11] Ding, L., Tan, J., Feng, Y., Gao, Y. (2009). Multi-objective optimization for disassembly line balancing based on Pareto ant colony algorithm, Computer Integrated Manufacturing System, Vol. 15, 1406-1413, doi: 10.13196/ j.cims.2009.07.160.dinglp.005.

[12] Cao, J., Xia, X., Wang, L., Zhang, Z., Liu, X. (2019). A novel multi-efficiency optimization method for disassembly line balancing problem, Sustainability, Vol. 11, No. 24, Article No. 6969, doi: 10.3390/su11246969.

[13] Özceylan, E., Kalayci, C.B., Güngör, A., Gupta, S.M. (2019). Disassembly line balancing problem: A review of the state of the art and future directions, International Journal of Production Research, Vol. 57, No. 15-16, 4805-4827, doi: $10.1080 / 00207543.2018 .1428775$.

[14] Deniz, D., Ozcelik, F. (2019). An extended review on disassembly line balancing with bibliometric \& social network and future study realization analysis, Journal of Cleaner Production, Vol. 225, 697-715, doi: 10.1016/ j.jclepro.2019.03.188.

[15] Gupta, S.M., Erbis, E., McGovern, S.M. (2019). Disassembly sequencing problem: A case study of a cell phone. In: Proceedings of Conference on Environmentally Conscious Manufacturing IV, Philadelphia, Pennsylvania, USA, doi: $10.1117 / 12.577196$.

[16] Xia, X., Liu, W., Zhang, Z., Wang, L., Cao, J., Liu, X. (2019). A balancing method of mixed-model disassembly line in a random working environment, Sustainability, Vol. 11, No. 8, Article No. 2304, doi: 10.3390/su11082304.

[17] Song, S., Zhang, W., Zhang, L. (2016). Product disassembly sequence planning based on improved artificial bee colony algorithm, China Mechanical Engineering, Vol. 27, No. 17, 2384-2390, doi: 10.3969/j.issn.1004-132X.2016. $\underline{17.019}$.

[18] Yu, B., Wu, E., Chen, C., Yang, Y., Yao, B.Z., Lin, Q. (2017). A general approach to optimize disassembly sequence planning based on disassembly network: A case study from automotive industry, Advances in Production Engineering \& Management, Vol. 12, No. 4, 305-320, doi: 10.14743/apem2017.4.260.

[19] Lv, Y., Zhang, J., Qin, W. (2017). A genetic regulatory network-based sequencing method for mixed-model assembly lines, Advances in Production Engineering \& Management, Vol. 12, No. 1, 62-74, doi: 10.14743/ apem 2017.1.240.

[20] Yang, M.S., Ba, L., Liu, Y., Zheng, H.Y., Yan, J.T., Gao, X.Q., Xiao, J.M. (2019). An improved genetic simulated annealing algorithm for stochastic two-sided assembly line balancing problem, International Journal of Simulation Modelling, Vol. 18, No. 1, 175-186, doi: 10.2507/IJSIMM18(1)C04.

[21] Wang, Y., Yang, 0. (2017). Research on industrial assembly line balancing optimization based on genetic algorithm and witness simulation, International Journal of Simulation Modelling, Vol. 16, No. 2, 334-342, doi: 10.2507/IJSIMM16(2)C08.

[22] Suszyński, M., Żurek, J., Legutko, S. (2014). Modeling of assembly sequences using hypergraph and directed graph, Tehnički Vjesnik - Technical Gazette, Vol. 21, No. 6, 1229-1233.

[23] Holland, J.H. (1975). Adaptation in natural and artificial systems, The University of Michigan Press, MIT Press, Michigan, USA, 28-35.

[24] Su, Y., Zhang, Z., Hu, Y. (2016). A variable neighborhood search algorithm for disassembly line balancing problem, Modern Manufacturing Engineering, Vol. 10, 19-23, doi: 10.16731/j.cnki.1671-3133.2016.10.005.

[25] Zhang, J., Fang, J.B., Gao, L. (2015). Disassembly sequence planning based on quantum genetic algorithm, Modern Manufacturing Engineering, Vol. 4, 110-115, doi: 10.16731/j.cnki.1671-3133.2015.04.013.

[26] Seidi, M., Saghari, S. (2016). The balancing of disassembly line of automobile engine using genetic algorithm in fuzzy environment, Industrial Engineering and Management Systems, Vol. 15, No. 4, 364-373, doi: 10.7232/iems. 2016.15.4.364.

[27] Zhang, X.F., Wei, G., Guo, Y.R., Hu, Z.Y. (2014). Study on change response performance of disassembly model for design for disassembly, Mechanical Design and Manufacturing, Vol. 1, 251-253, doi: 10.19356/i.cnki.1001-3997. 2014.01.077.

[28] Guo, H., Liang, J., Zhang, S. (2015). Optimization and examples in Matlab GA toolbox GADS, Electronic Design Engineering, Vol. 23, No. 10, 27-32, doi: 10.14022/i.cnki.dzsigc.2015.10.009.

[29] McGovern, S.M., Gupta, S.M. (2007). A balancing method and genetic algorithm for disassembly line balancing, European Journal of Operational Research, Vol. 179, No. 3, 692-708, doi: 10.1016/i.ejor.2005.03.055.

[30] Li, Y. (2015). Automobile engine structure and disassembly, Beijing Institute of Technology press, Beijing, China. 


\title{
Optimizacija uravnoteženja demontažne linije z uporabo izboljšanega večkriterijskega genetskega algoritma
}

\author{
Wang, Y.J. ${ }^{a}{ }^{*}$, Wang, N.D. ${ }^{a}$, Cheng, S.M. ${ }^{a}$, Zhang, X.C. ${ }^{a}$, Liu, H.Y. ${ }^{a}$, Shi, J.L. ${ }^{a}$, Ma, Q.Y. ${ }^{a}$, Zhou, M.J. ${ }^{a}$ \\ aschool of Mechanical Engineering and Automation, Dalian Polytechnic University, P.R. China
}

\section{POVZETEK}

Demontaža je prisotna $\mathrm{v}$ različnih postopkih obnove, vključno s ponovno izdelavo, recikliranjem in odstranjevanjem. Demontaža izdelka je učinkovit način recikliranja odpadkov in je nujen pogoj za krožen življenjski cikel izdelka. Glede na značilnosti demontažne linije, ki temelji na zmanjševanju števila delovnih postaj in uravnoteženju časa nedejavnosti postaje, so kazalnik škodljivosti, kazalnik povpraševanja in število sprememb smeri predlagani kot novi cilji optimizacije. Na podlagi analize tradicionalnega genetskega algoritma ta članek obravnava problem uravnoteženja demontažne linije kot večkriterijski. Problem uravnoteženja demontažne linije spada med NP-težke probleme. Inteligentni optimizacijski algoritmi kažejo odlične lastnosti pri reševanju takšnih problemov. Glede na značilnosti tradicionalne metode reševanja problema uravnoteženja demontažne linije, kjer je bil rezultat rešitve en sam in ni mogel izpolniti številnih meril uravnoteženja, je bil za rešitev modela predlagan izboljšan večkriterijski genetski algoritem s hitrejšo konvergenco. Na podlagi primera demontaže, je bil izboljšan večkriterijski genetski algoritem v primerjavi z obstoječim hevrističnim algoritmom z enim samim ciljem potrjen kot učinkovit in izvedljiv ter je bil uporabljen na dejanskem primeru demontaže. Podani sta dve študiji primera, in sicer postopek demontaže avtomobilskega motorja in demontaža računalniških komponent.

\section{PODATKI O ČLANKU}

Ključne besede:

Montaža;

Demontaža;

Uravnoteženje linje;

Večkriterijska optimizacija;

Ponovna izdelava;

Obnova izdelka;

Življenjski cikel izdelka;

NP-težki problemi;

Izboljšan genetski algoritem

*Kontaktna oseba:

wangyj@dlpu.edu.cn

(Wang, Y.J.)

Zgodovina članka:

Prejet 13. maja 2021

Popravljen 23. maja 2021

Sprejet 4. junija 2021

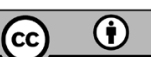

Content from this work may be used under the terms of the Creative Commons Attribution 4.0 International Licence (CC BY 4.0). Any further distribution of this work the work, journal citation and DOI 